\title{
Irigenin exerts anticancer effects on human liver cancer cells via induction of mitochondrial apoptosis and cell cycle arrest
}

\author{
Wanggang Xu, Yingmin Kuang, Dan Wang, Zhen Li and Renpin Xia*
}

\begin{abstract}
Irigenin has been reported to exhibit remarkable anticancer effects against several human cancers. Nonetheless, the anticancer effects of irigenin against the human liver cancer cells are still largely unexplored. Consistently, this study was designed to evaluate the anticancer effects of irigenin against human liver cancer cells and to unveil the underlying molecular mechanisms. The results showed that irigenin significantly $(p<0.05)$ inhibited the growth of the human HepG2 and SNU-182 liver cancer cells with an IC 50 value of $14 \mu \mathrm{M}$. Nonetheless, the cytotoxic effects of irigenin against the normal THLE-2 cells were comparatively lower as evident from the $I \mathrm{C}^{50}$ of $120 \mu \mathrm{M}$. The AO/EB and DAPI staining showed that irigenin induces apoptosis in the human liver cancer cells. Annexin V/PI staining assay revealed a significant $(p<0.05)$ increase in the percentage of apoptotic HepG2 and SNU-182 liver cancer cells upon treatment with irigenin. It was found that the number of apoptotic HepG2 and SNU-182 cells enhanced from 2.3 to $41.75 \%$ and 1.16 to $51.9 \%$ at IC50, respectively. Western blot showed a considerable increase in Bax and decrease in the BCl-2 expression upon irigenin treatment further confirming the induction of apoptosis. Flow cytometric analysis revealed that irigenin also induces G2/M cell cycle arrest of HepG2 and SNU-182 cells. The percentage of G2/M phase HepG2 and SNU-182 cells increased from 17.92 to $34.35 \%$ and 23.97 to $38.23 \%$ at $I C_{50}$, respectively This was also accompanied by decrease in the expression of CDK1 and Cyclin-B in HepG2 and SNU-182 cells. Taken together, the results of the present study suggest that irigenin inhibits the growth of the human liver cancer cells via induction of apoptosis and cell cycle arrest. These results point towards the potential of irigenin as a lead for the development of chemotherapy for liver cancer.
\end{abstract}

Keywords: Liver cancer, Isoflavonoids, Irigenin, Cell cycle, Apoptosis

\section{Introduction}

Liver cancer is a disastrous human malignancy and a leading cause of mortality in several regions of the world $[10,11]$. World has seen huge upsurge in liver cancer incidences from past two decades. It has been ranked fourth among highest mortality causing cancers with more than 750,000 cases diagnosed annually [19]. Over $80 \%$ of liver cancer cases befall in middle and low-resource countries, predominantly in sub-Saharan

*Correspondence: 81241719@qq.com

Department of Organ Transplantation, The First Affiliated Hospital

of Kunming Medical University, Kunming 650032, Yunnan, China
Africa and Eastern Asia, where social and health care systems are mostly constrained. Major risk factors that contribute to liver cancer development includes chronic hepatitis B and C, metabolic liver disease (MLD, nonalcoholic fatty liver disease, alcohol consumption and exposure to dietary toxins like aristolochic acid and aflatoxins [7, 22]. Liver cancer surveillance is imperative for high risk patients which permits early disease detection and boosts the efficacy of curative methodologies. Curative treatments involved in early-stage liver cancer include surgical resection, local ablation, chemotherapy, and organ transplantation. Regardless of substantial advancements made in liver cancer diagnosis and treatment, the overall survival remains extremely 
poor with high frequency of disease recurrence [18]. Therefore, to address the rising global liver cancer burden, potential treatment methodologies and chemopreventive drugs are need of the hour. Natural products have impressed the researchers with their enormous structural diversity and bioactivity profiles [3]. More than $70 \%$ of chemopreventives for cancer belong to either natural products or compounds derived from them [6]. Isoflavonoids are a major class of natural products and exhibit several bioactivities such as antiproliferative, anticarcinogenic, antimutagenic and antioxidant activities $[4,15,17]$. Irigenin is one of the active isoflavonoids present in several plant species [9]. Irigenin has been shown to exert anticancer effects on breast MBD-MA-231 cancer cells by inducing apoptosis. These proapoptotic effects of irigenin were reported to interceded via reactive-oxygen species (ROS) mediated mitochondrial dysfunction [24]. Irigenin has been reported to suppress prostaglandin (PG) E2 production, lipopolysaccharide (LPS)-induced nitric oxide (NO) production, NF- $\mathrm{kB}$ activity and expression levels of p-65 protein, in murine RAW 264.7 macrophages [1]. Furthermore, it has been shown to exhibit substantial inhibitory effects on lung cancer metastasis induced via Fibronectin-Extra Domain A [2]. In yet another study irigenin has been reported to possesses promising TRAIL (Tumor necrosis factor-related apoptosisinducing ligand) based proapoptotic effects against gastric cancer [20]. However, the anticancer effects of irigenin against liver cancer and its underlying mechanism is still largely unknown. Against this backdrop, the anticancer effects of irigenin were examined against liver cancer HepG2 and SNU-182 cells in vitro and the potential underlying mechanisms were explored.

\section{Materials and methods}

\section{Reagents, chemicals, and cell cultures}

Irigenin (98\% purity, CAS Number: 548-76-5) was obtained from Sigma-Aldrich (St. Louis, Missouri, United States). Other chemicals involved in this research were bought from Merck KGaA (Darmstadt, Germany), unless otherwise mentioned. The HepG2 and SNU-182 liver cancer and THLE-2 normal liver cells were bought from Type Culture Collection of Chinese Academy of Sciences, Shanghai, China. RPMI-1640 media (Hyclone, Logan, UT) was used for seeding all the cell culture maintaining fetal bovine serum (FBS; 10\%) (Thermo Fisher Scientific, Inc., Waltham, United States), penicillin $\mathrm{G}(100 \mathrm{U} / \mathrm{ml})$ and streptomycin $(100 \mu \mathrm{g} / \mathrm{ml})$ as potential antibiotics. Humid environment of $5 \% \mathrm{CO}_{2}$ concentration and $37^{\circ} \mathrm{C}$ was used to maintain and culture all-inclusive cell lines.

\section{Proliferation assay}

The effects of irigenin treatment on proliferation rate of HepG2 and SNU-182 liver cancer and THLE-2 normal liver cells were monitored by WST-1 viability assay as described previously $[10,11]$. Briefly, HepG2, SNU182 and THLE-2 cell lines were precultured for $24 \mathrm{~h}$ followed by transference to 96 -well plates at a concentration of $1.5 \times 10^{5}$ cells per well. Afterwards, irigenin was added to each well plate at altering concentrations ranging from 0 to $640 \mu \mathrm{M}$. After $48 \mathrm{~h}$ of treatment, each cell line was washed using phosphate buffered saline (Sigma) and then subjected to $3 \mathrm{~h}$ of incubation with WST-1 $(10 \mu \mathrm{L})$ at $37^{\circ} \mathrm{C}$. DMSO (Sigma) was used as vehicle control. Finally, viability was determined by taking optical density (OD) at $450 \mathrm{~nm}$ using ELISA microplate reader (BioTek, China). The absorbance of untreated HepG2, SNU-182 and THLE-2 cells (control) were considered as $100 \%$ viable and absorbance was also considered as $100 \%$. The experiments were performed in triplicate and the percentage inhibition was calculated by using the given formula:

$$
\begin{aligned}
& \text { Inhibition }(\%) \\
& \quad=100-\frac{\text { OD of treated cells }}{\text { OD of untreated control cells }} \times 100
\end{aligned}
$$

\section{Apoptosis assays}

To determine apoptotic effects of irigenin against HepG2 and SNU-182 liver cancer cells Acridine Orange/Ethidium Bromide (AO/EB) staining, 4,6-diamidino-2-phenylindole staining (DAPI) staining were performed as described previously [23]. For $\mathrm{AO} / \mathrm{EB}$ staining, both the cancer cell lines were seeded in 6-well plates containing RPMI1604 culture medium (Hyclone, Logan, UT) at the density of $0.4 \times 10^{6}$ cells each well. Afterwards, cells were precultured for $12 \mathrm{~h}$ prior to irigenin exposure at doses of 0 and $14 \mu \mathrm{M}$ at $37{ }^{\circ} \mathrm{C}$ for $24 \mathrm{~h}$. Post treatment, as the cells start to slough off, $25 \mu \mathrm{l}$ of cell culture was loaded to glass slides. Thereafter, $1 \mu \mathrm{l}$ of AO $(100 \mu \mathrm{g} /$ $\mathrm{ml})$ and $\mathrm{EB}(100 \mu \mathrm{g} / \mathrm{ml})$ staining solution was added to each slide followed by covering with coverslips. Finally, these slides were placed under a fluorescence microscope (Nikon Inc., Japan) for investigation of apoptotic effects. For DAPI staining assay, cells were cultured and treated with irigenin same as for $\mathrm{AO} / \mathrm{EB}$ staining assay. Following irigenin treatment, cells were washed with PBS and then fixed within $10 \%$ of formaldehyde. Post fixation cells were again washed with PBS and then stained with DAPI $(1 \mu \mathrm{g} / \mathrm{ml})$ for $25 \mathrm{~min}$ in dark. Finally, DAPI stained cells were loaded to a fluorescence microscope (Nikon Inc., Japan) for apoptosis assessment. Annexin V/PI staining assays were performed in 
accordance with previously described procedures [12]. The experiments were performed in triplicate.

\section{Flow cytometry}

Flow cytometry was performed to check the effects of irigenin on different cell cycle check points in HepG2 and SNU-182 liver cancer cells [8]. Both the cancer cell lines at a density of $1 \times 10^{4}$ cells/well were incubated for $12 \mathrm{~h}$ prior to their exposure with variant irigenin doses $(0-320 \mu \mathrm{M})$ for a time period of $24 \mathrm{~h}$. Post irigenin treatment, cells were subjected to washing with PBS three times followed by PI staining. Finally, the distribution of irigenin treated cells in different cell cycle phase was monitored by flow cytometry using a FACS flow cytometer (BD FACSCalibur, BD Biosciences, USA). The experiments were performed in triplicate.

\section{Western blotting}

Western blotting was performed to examine the expression of proteins in in irigenin treated HepG2 and SNU-182 liver cancer cells as described previously [8]. In brief, both the cell lines were treated with different irigenin doses $(0$ and $14 \mu \mathrm{M})$ for $48 \mathrm{~h}$ at a concentration of $1.5 \times 10^{4}$ cells/well of 24 -well plate. Treated HepG2 and SNU-182 cells were then lysed incubating the cells with RIPA lysis buffer (Sigma) on ice for $20 \mathrm{~min}$. Each lysate was subjected to BCA assay for protein quantification. About $40 \mu \mathrm{g}$ of proteins from each lysate were separated using $10 \%$ SDS-PAGE separation followed by electrophoretic transference to nitrocellulose membranes (Beijing Dingguo Changsheng Biotechnology Co., Ltd.). These membranes were blocked by nonfat milk followed by subjecting to primary antibody treatment overnight at $4{ }^{\circ} \mathrm{C}$ using antibodies against Bax (Cat\# sc-20067), Bcl-2 (Cat\# sc-517390), CDK1 (Cat\# sc-53217), Cyclin-B (Cat\# sc-166210), CyclinA (Cat\# sc-239), Cyclin-D (Cat\# sc-53271), Cyclin-E (Cat\# sc-377100), Actin (Cat\# sc-58673) (all 1:1,000; Santa Cruz Biotechnology, Inc., Dallas, United States). Afterwards, membranes were then exposed to $1 \mathrm{mg} /$ $\mathrm{mL}$ horse radish peroxidase-conjugated anti-rabbit secondary antibody (Cat\# sc-2372) (1:10,000 dilutions, Santa Cruz Biotechnology, Inc., Dallas, United States). Finally, the protein signals were recorded with the help of ECL Detection kit (GE Healthcare Life Sciences, Sweden).

\section{Statistical analysis}

One-Way Analysis of Variance was used to carry out statistical analysis and the significance level was tested at $p<0.05$. All the data from independent triplicate experiments was expressed as mean \pm SD. GraphPad Prism software (version 7; GraphPad Software, Inc., La Jolla, CA, USA) was used for statistical analysis.

\section{Results}

Irigenin inhibited proliferation of HepG2 and SNU-182 liver cancer cells

The cytotoxic effects of irigenin (Fig. 1a) were investigated against HepG2 and SNU-182 liver cancer and THLE-2 normal liver cells using WST-1 assay. The results showed a significant $(p<0.05)$ decrease of HepG2 and SNU-182 cell proliferation upon treatment with different concentrations of irigenin (Fig. 1b). Irigenin induced dose-dependent cytotoxicity against HepG2 and SNU182 cells and exhibited an $\mathrm{IC}_{50}$ of $14 \mu \mathrm{M}$. Nonetheless, the cytotoxic effects of irigenin against the normal THLE- 2 were found to be comparatively less as evident from an $\mathrm{IC}_{50}$ of $120 \mu \mathrm{M}$.

\section{Irigenin induced apoptotic cell death in HepG2 and SNU-182 liver cancer cells}

Herein, we investigated the proapoptotic related antiproliferative effects of irigenin against HepG2 and SNU-182

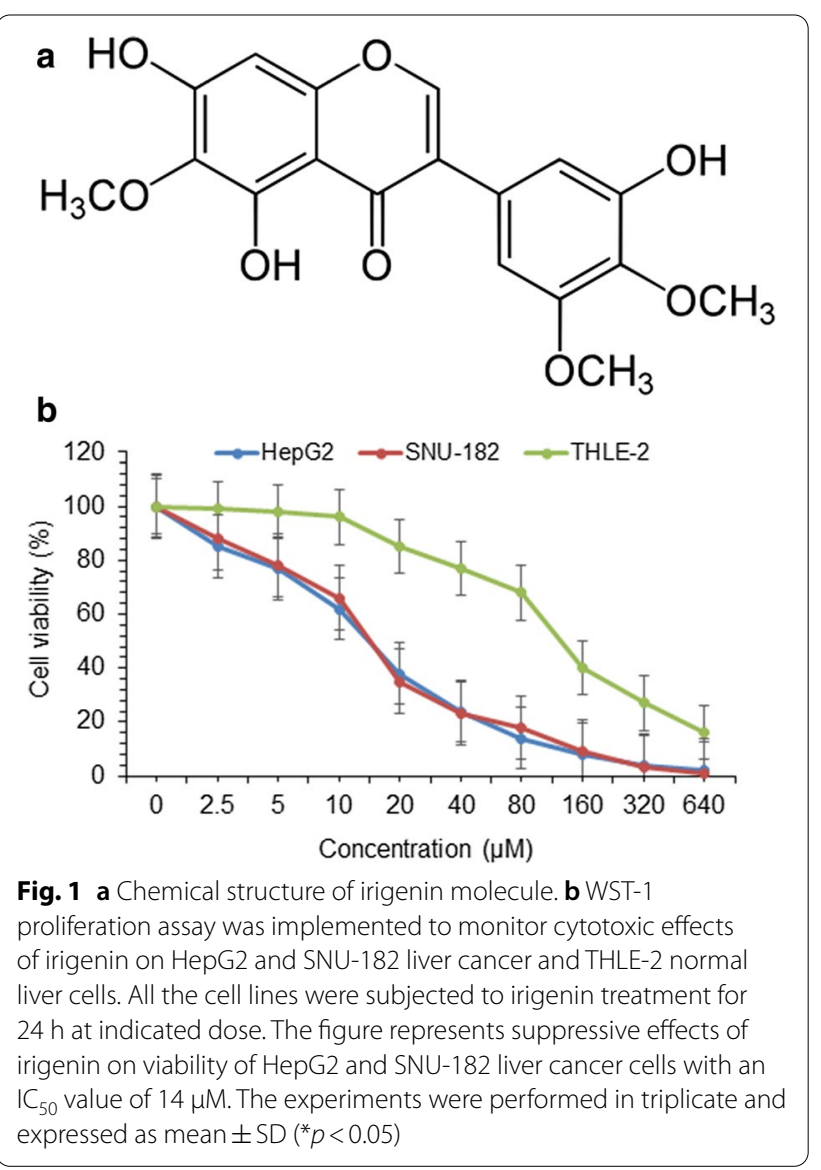




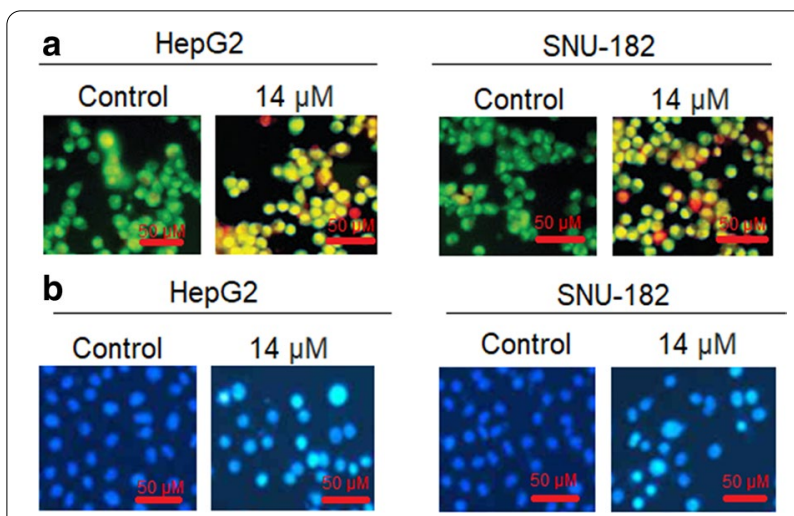

Fig. 2 a AO/EB staining assay was executed to monitor apoptosis in irigenin treated HepG2 and SNU-182 cells using florescence microscopy. The figure depicts green fluorescence in control group while as yellow-green, orange-red and red florescence (showing early, late apoptotic and necrotic cells) in irigenin treated group. b DAPI staining assay results showing white nuclei (apoptotic nuclei) in irigenin treated group as compared to control group. The experiments were performed in triplicate

cells. AO/EB staining results showed that irigenin induced apoptosis in HepG2 and SNU-182 cell lines in a dose-reliant manner. In control group only green fluorescence could be seen, marking normal cells, while in treated group yellow-green, orange-red, and red florescence was observed, marking apoptotic (early and late stage) and necrotic cells (Fig. 2a). Further, DAPI staining showed white colored nuclei in irigenin treated group as compared to control group indicative of apoptotic cell death in HepG2 and SNU-182 cells (Fig. 2b). Furthermore, annexin V/PI dual staining revealed a significant $(p<0.05)$ increase in the percentage of the apoptotic HepG2 and SNU-182 cells. It was found that the number of apoptotic HepG2 and SNU-182 cells enhanced from 2.3 to $41.75 \%$ and 1.16 to $51.9 \%$ at $\mathrm{IC}_{50}$, respectively (Fig. 3a). The antiproliferative effects of irigenin were further supported by western blotting. Results detected remarkable increase in the expression of Bax proteins and decrease in expression of Bcl-2 (Fig. 3b). Therefore, it may be concluded that antiproliferative effects of irigenin are mediated via mitochondrial apoptosis in HepG2 and SNU-182 cancerous liver cells.

\section{Irigenin induced cell cycle arrest in HepG2 and SNU-182 liver cancer cells}

The effects of irigenin on cell cycle progression were assessed by flow cytometric analysis. After exposure of HepG2 and SNU-182 cells to different irigenin doses

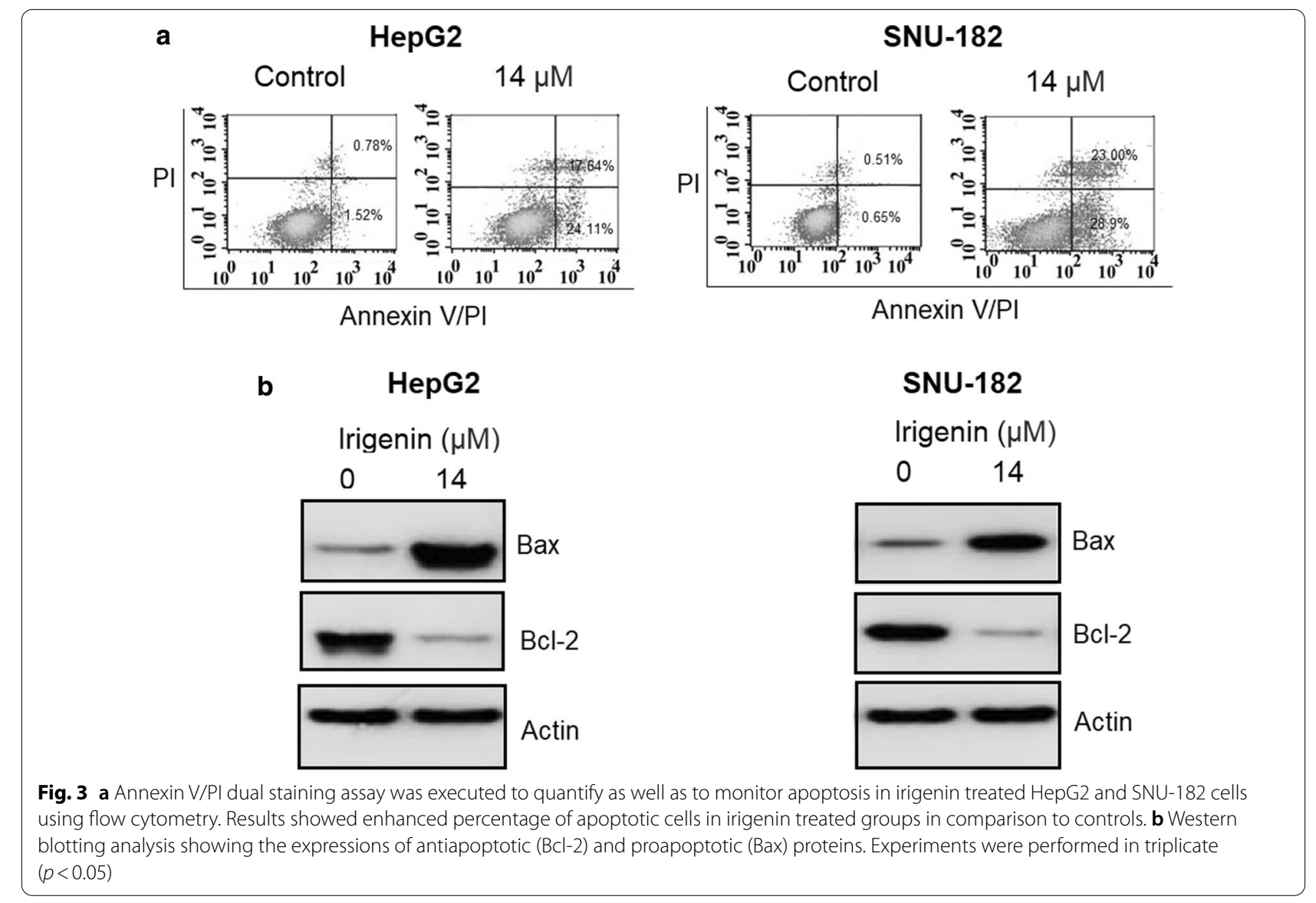


(0-320 $\mu \mathrm{M})$, staining with PI was performed prior to flow cytometric assessment. Results showed that the percentage of G2/M phase HepG2 and SNU-182 cells increased from 17.92 to $34.35 \%$ and 23.97 to $38.23 \%$ at $\mathrm{IC}_{50}$, respectively (Fig. 4a). These results indicated that irigenin inhibited the cell cycle progression at G2/M phase. Moreover, western blotting showed that irigenin could potentially downregulate the expressions of important cell cycle associated proteins including CDK1 and Cyclin-B in HepG2 and SNU-182 cells (Fig. 4b). The expression of Cyclin A, D and E remained apparently unchanged.

\section{Discussion}

Unfortunately, there is an alarming increase in the number of liver cancer incidences and deaths, globally. Despite of improvements have been made in diagnosis, monitoring and treatment, it remains one of the biggest challenges for researchers. Growing alcohol abuse and unprecedented upsurge of obesity in developing as well as developed countries is an issue of major concern in growing global liver cancer burden [21]. The presently accessible treatment options of liver cancer possess potential shortcoming like disease relapse, lethal side effects and poor survival rates, except being opted at early stages. Therefore, there is a scarcity of standard and capable chemopreventives which can produce efficient results against liver cancer. The current investigation was instigated to unveil the anticancer effects of irigenin isoflavonoid against liver cancer. Irigenin molecule has been reported of remarkable biological and pharmacological potential [2]. Irigenin has been earlier reported of strong antiproliferative effects against several human cancer cell including prostate cancer, gastric cancer and breast cancer [1, 16, 24]. It was reported that irigenin has a remarkable tendency to target key survival signalling pathways in cancer cells like apoptosis. Herein, results showed that irigenin could potentially inhibit the proliferation of both HepG2 and SNU-182 cell lines in a concentration-reliant manner. It showed an $\mathrm{IC}_{50}$ value of $14 \mu \mathrm{M}$ against both the target cancer cell lines. The similar trend of cell viability reduction in both HepG2 and SNU-182 cells could be due to their similar origin (hepatocellular carcinoma

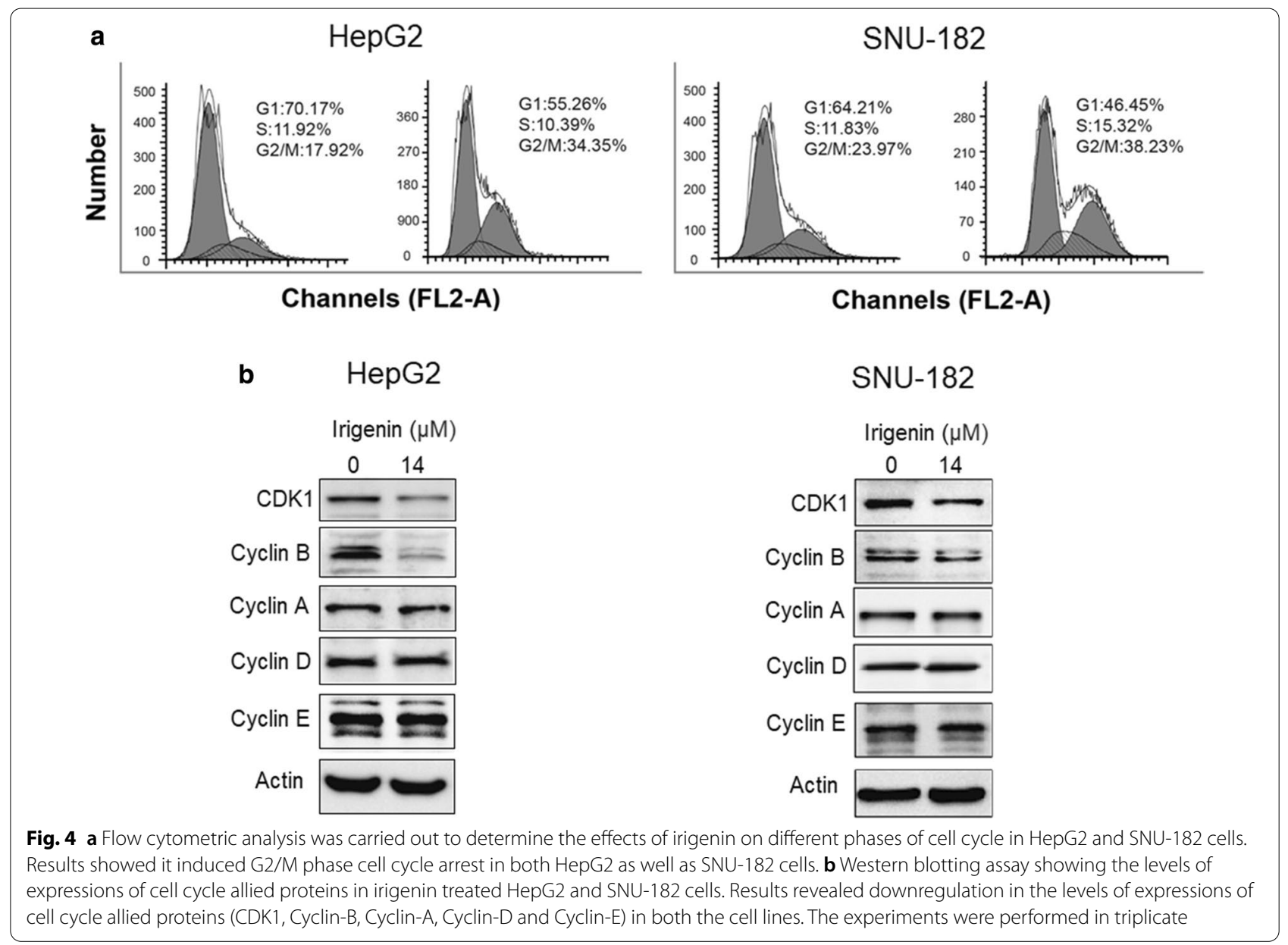


cell lines). Moreover, the antiproliferative effects of irigenin on the normal THLE-2 cells was comparatively lower as evident from a very high $\mathrm{IC}_{50}(120 \mu \mathrm{M})$. Nonetheless, irigenin at higher doses causes drastic reduction in the viability of the normal cells as well. This could be due to several reasons, for instances, at higher concentrations irigenin might interfere with several cellular enzymes leading to increased cytotoxicity. It is noteworthy, that the $\mathrm{IC}_{50}$ of irigenin against the liver is around 9 times lower than the normal cells and as such irigenin should be considered as an important lead molecule against liver cancer. Next, efforts were made to unveil the underlying mechanism of action of antiliver cancer effects of irigenin. Apoptosis is one of the leading targets of anticancer drugs and occurs naturally in multicellular organisms to discard damaged, aged, and malfunctioning cells [5]. Apoptosis gets stimulated by intrinsic (mitochondrial mediated) and extrinsic (death receptors) factors. Irigenin has been previously reported of substantial proapoptotic effects against gastric cancer cells through upregulation of PARP, Caspase-3, -8 and -9 , and Bax activities [20]. Herein, the apoptotic effects of irigenin against HepG2 and SNU182 cells were modulated by increase in Bax expression levels and decrease in Bcl-2 expression levels. These results were similar to previously reported for irigenin against gastric cancer cells and breast cancer cells [20, 24]. Therefore, irigenin induced cytotoxic effects in HepG2 and SNU-182 cell lines may be attributed to its proapoptotic effects.

Further, uncontrolled, random and frequent cell division is one of the primary features of malignant cancers [13]. Cell division commences through different check points (S, G0/G1 and G2/M) during its course of completion [14]. Chemopreventive drugs target these check points and block the progression of cell cycle at any of the stages. Irigenin has been reported of remarkable potential to inhibit the cell cycle progression in different human cancer cell lines including PC3, RWPE-1 and LNCaP prostate cancer cells at G1-phase [16]. Herein, irigenin induced $G_{2} / M$ phase cell cycle arrest of both HepG2 and SNU-182 cell lines via downregulation of CDK1 and Cyclin-B. The CDK1 and cyclin B are the main regulates of $G_{2} / M$ check point [14]. Consistently, irigenin inhibits the expression of CDK1 and cyclin $B$ to trigger $\mathrm{G}_{2} / \mathrm{M}$ cell cycle arrest. In contrary, CyclinA, Cyclin-D and Cyclin-E play roles in other phases of the cell lines and as such their expression remains more or less unaltered. Therefore, the property of inducing cell cycle arrest by irigenin is parallel to previously reported data where irigenin drug induced cell cycle arrest by modulating the expressions of key proteins in prostate cancer cells [16]. Taken together, the results of this investigation indicate that irigenin could potentially suppress human liver cancer. The anti-liver cancer potency of irigenin was observed to mediate via induction of mitochondrial apoptosis and cell cycle arrest. Therefore, irigenin may prove a lead drug in liver cancer management and research.

\section{Acknowledgements \\ All the author of this manuscript is thankful to The First Affiliated Hospital of Kunming Medical University, Kunming , Yunnan, China to conduct the presented protocol.}

\section{Authors' contributions}

WX and RX conceived the idea and designed the study. WX, YK, DW and ZL performed the experiments and collect the data. WX, YK and RX were involved in the statistical analysis. RX supervised the work and drafted the manuscript. All authors read and approved the final manuscript.

\section{Funding}

This study was supported by Yunnan Provincial Department of Science and Technology_Kunming Medical University Joint Project on Application Foundation (No: 2017FE467(047)).

\section{Availability of data and materials}

Not applicable.

Ethics approval and consent to participate

Not applicable.

\section{Consent for publication}

Not applicable.

\section{Competing interests}

The authors declare no competing interests.

Received: 13 August 2020 Accepted: 25 November 2020

Published online: 23 January 2021

\section{References}

1. Ahn KS, Noh EJ, Cha KH, Kim YS, Lim SS, Shin KH, Jung SH (2006) Inhibitory effects of Irigenin from the rhizomes of Belamcanda chinensis on nitric oxide and prostaglandin E2 production in murine macrophage RAW 264.7 cells. Life Sci 78:2336-2342

2. Amin A, Chikan NA, Mokhdomi TA, Bukhari S, Koul AM, Gharemirshamlu SBA, FR, Wafai AH, Qadri A, Qadri RA, (2016) Irigenin, a novel lead from Western Himalayan chemiome inhibits Fibronectin-Extra Domain A induced metastasis in Lung cancer cells. Sci Rep 6:37151

3. Baba SA, Malik AH, Wani ZA, Mohiuddin T, Shah Z, Abbas N, Ashraf N (2015) Phytochemical analysis and antioxidant activity of different tissue types of Crocus sativus and oxidative stress alleviating potential of saffron extract in plants, bacteria, and yeast. S Afr J Bot 201(99):80-87

4. Birt DF, Hendrich S, Wang W (2001) Dietary agents in cancer prevention: flavonoids and isoflavonoids. Pharmacol Ther 90:157-177

5. Carballo GB, Ribeiro JH, de Faria Lopes GP, Ferrer VP, Dezonne RS (2020) Pereira CM (2020) GANT-61 Induces Autophagy and Apoptosis in Glioblastoma Cells despite their heterogeneity. Cell Mol Neurobiol. https:// doi.org/10.1007/s10571-020-00891-6

6. Demain AL, Vaishnav P (2011) Natural products for cancer chemotherapy. Microbial biotechnol 4(6):687-699

7. El-Serag HB (2012) Epidemiology of viral hepatitis and hepatocellular carcinoma. Gastroenterology 142:1264-1273

8. Kang X, Wang H, Li Y, Xiao Y, Zhao L, Zhang T, Zhou S, Zhou X, Li Y, Shou Z, Chen C (2019) Alantolactone induces apoptosis through ROS-mediated AKT pathway and inhibition of PINK1-mediated mitophagy in human HepG2 cells. Artif Cells Nanomed Biotechnol 47(1):1961-1970

9. Lee SO, Woo WS, Woo EH, Kim KS (1989) Isoflavonoids of Belamcanda chinensis. Saengyak Hakhoe Chi 20:219-222 
10. Liu JH, Yang Y, Song Q, Li JB (2019) MicroRNA-155 regulates the proliferation and metastasis of human breast cancers by targeting MAPK7. J BUON 24:1075-1080

11. Liu Z, Jiang Y, Yuan H, Fang Q, Cai N, Suo C, Jin L, Zhang T, Chen X (2019) The trends in incidence of primary liver cancer caused by specific etiologies: results from the Global Burden of Disease Study 2016 and implications for liver cancer prevention. J Hepatol 70(4):674-683

12. Lukanova A, Kaaks R (2005) Endogenous hormones and ovarian cancer: epidemiology and current hypotheses. Cancer Epidemiol Biomarkers Prev 14:98-107

13. Man SM, Zhu Q, Zhu L, Liu Z, Karki R, Malik A, Sharma D, Li L, Malireddi RS, Gurung P, Neale G (2015) Critical role for the DNA sensor AIM2 in stem cell proliferation and cancer. Cell 162:45-58

14. Matellán L, Monje-Casas F (2020) Regulation of mitotic exit by cell cycle checkpoints: lessons from Saccharomyces cerevisiae. Genes 11:195

15. Miadokova E, Masterova I, Vlckova V, Duhova V, Toth J (2002) Antimutagenic potential of homoisoflavonoids from Muscari racemosum. J Ethnopharmacol 18:381-386

16. Morrissey C, Bektic J, Spengler B, Galvin D, Christoffel V, Klocker H, Fitzpatrick JM, Watson RW (2004) Phytoestrogens derived from Belamcanda chinensis have an antiproliferative effect on prostate cancer cells in vitro. J Urol 172:2426-2433

17. Ryan-Borchers TA, Park JS, Chew BP, McGuire MK, Fournier LR, Beerman KA (2006) Soy isofl avones modulate immune function in healthy postmenopausal women. Am J Clin Nutr 83:118-1125
18. Serper M, Taddei TH, Mehta R, D'Addeo K, Dai F, Aytaman A, Baytarian M Fox R, Hunt K, Goldberg DS, Valderrama A (2017) Association of provider specialty and multidisciplinary care with hepatocellular carcinoma treatment and mortality. Gastroenterology 152:1954-1964

19. Villanueva A (2019) Hepatocellular carcinoma. N Engl J Med 380:1450-1462

20. Xu Y, Gao CC, Pan ZG, Zhou CW (2018) Irigenin sensitizes TRAlL-induced apoptosis via enhancing pro-apoptotic molecules in gastric cancer cells. Biochem Biophys Res Commun 496:998-1005

21. Yang JD, Hainaut P, Gores GJ, Amadou A, Plymoth A, Roberts LR (2019) A global view of hepatocellular carcinoma: trends, risk, prevention and management. Nat Rev Gastroenterol Hepatol 22:1-6

22. Yang JD, Roberts LR (2010) Hepatocellular carcinoma: a global view. Nat Rev Gastroenterol Hepatol 7:448-458

23. Yin Z, Yi J, Nie T, Yang Z, Ding N, Du SX, Liu S, Peng T (2019) Silencing of PPMD1 inhibits proliferation of human colon cancer cells via induction of apoptosis and cell cycle arrest. J BUON 24(4):1464-1469

24. Zhi X, Li C, Liu J, Ji Y (2019) Irigenin from Belamcanda chinensis (L.) DC induces apoptosis of human breast cancer MDB-MA-231 cells via mitochondria-dependent pathway. Lat Am J Pharm 38:13-17

\section{Publisher's Note}

Springer Nature remains neutral with regard to jurisdictional claims in published maps and institutional affiliations.

\section{Submit your manuscript to a SpringerOpen ${ }^{\circ}$ journal and benefit from:}

- Convenient online submission

- Rigorous peer review

- Open access: articles freely available online

- High visibility within the field

- Retaining the copyright to your article

Submit your next manuscript at $\boldsymbol{\Delta}$ springeropen.com 